\title{
LA TÉCNICA DE INCIDENTES CRÍTICOS: UNA HERRAMIENTA CLÁSICA Y VIGENTE EN ENFERMERÍA ${ }^{1}$
}

\author{
THE CRITICAL INCIDENTS TECHNIQUE: \\ A CLASSIC AND EFFECTIVE TOOL IN NURSING
}

\author{
ROdRIGo YÁÑEz G. * \\ LUIS LÓPEZ-MENA* \\ FERNANDO REYES R.**
}

\begin{abstract}
RESUMEN
Este artículo tiene como objetivo presentar una visión actualizada de la técnica de incidentes críticos, destacando que es una valiosa técnica de investigación cualitativa, flexible, relativamente fácil de aplicar, que permite comprender la interacción de las acciones de los trabajadores en el complejo contexto de la salud y es ampliamente utilizada en salud y en enfermería. En esta revisión se describe su origen, sus principales características, luego se explica cada una de las etapas básicas de su aplicación y también se analiza sus ventajas y desventajas. Finalmente, se discute su contribución a la investigación y su empleo en enfermería para evaluar acciones de intervención en centros de salud.
\end{abstract}

Palabras clave: Incidentes críticos, investigación cualitativa, metodología, confiabilidad, enfermería.

\begin{abstract}
This paper present an up-to-date vision of the critical incidents technique, highlighting that it is a valuable technique for qualitative investigation, flexible, of relative easy application and broadly used in nursing contexts. This technique also allows understanding the interaction between the actions of people in complex area of health context. In this review, its origin and main characteristics are described. Then a step-by-step explanation is given regarding the use of the technique, and also its advantages and disadvantages are analyzed. Finally, a discussion is presented of how it can contribute to research activities and to the assessment of intervention actions in health organizations.
\end{abstract}

Key words: Critical incident technique, qualitative research, methodology, reliability, nursing.

Fecha recepción: 10.05.10 Fecha aceptación: 11.05.11

\section{INTRODUCCIÓN}

En las últimas décadas se ha utilizado, cada vez con mayor frecuencia, la investigación cualitativa en distintas disciplinas, siendo cada vez más de creciente popularidad entre los investigadores en enfermería a través del mundo $(1,2)$. Si bien los resultados obtenidos con esta metodología de investi-

\footnotetext{
${ }^{1}$ Fuente de financiamiento: Proyecto Diuc Semilla 2005-172-010-1S, Universidad de Concepción, Chile.

* Psicólogo, Docente Departamento de Psicología, Facultad de Ciencias Sociales, Universidad de Concepción, Chile. E -mail: ryanez@udec.cl.

** Psicólogo. E-mail: hectormena@gmail.com.

${ }^{* * *}$ Psicólogo, Departamento de Psicología, Facultad de Ciencias Sociales, Universidad de Concepción, Chile. E-mail: fereyes@udec.cl
} 
gación son atractivos porque entregan una mayor comprensión dado que los datos son detallados y significativos, frecuentemente los investigadores señalan lo arduo que resulta el trabajo de recolectar, manejar, analizar e interpretar los datos. De este modo, se tiende a pensar que el uso de las técnicas cualitativas generalmente requiere una gran dedicación de tiempo en su puesta en práctica, dado que se genera un gran volumen de datos cuyo tratamiento es difícil tanto en la fase de recolección como en la de análisis (3). Esta situación podría indicar la necesidad de manejar técnicas de recolección y análisis de datos que resulten fáciles de utilizar, no sólo para investigadores profesionales y experimentados en salud, sino también para los mismos integrantes de estas comunidades de trabajo que llevan a cabo proyectos y requieren realizar estudio confiable en su contexto laboral (3). Por lo anterior, el presente trabajo tiene el propósito de presentar una visión actualizada de la técnica de incidentes críticos (Tics), técnica cualitativa con la que se puede, de forma eficiente, investigar en los centros de salud.

\section{ANTECEDENTES Y DEFINICIÓN DE LA TÉCNICA DE INCIDENTES CRÍTICOS}

La Tics fue desarrollada durante la Segunda Guerra Mundial por el psicólogo John C. Flanagan (1906-1996), quien enfrentó el desafío de mejorar el entrenamiento de los pilotos de guerra (4). Para ello desarrolló una metodología que le permitía describir con precisión los eventos o situaciones de éxito y fracaso en el trabajo de los pilotos $(5,6)$. Por ello, se puede considerar a Flanagan como el primero en realizar un esfuerzo sistemático por recolectar y analizar información a partir de incidentes específicos (7). A pesar que se empezó a utilizar esta técnica desde la década del 40, no fue sino hasta 1954 cuando Flanagan formalizó el proceso de recolección de datos y publicó un artículo en que la detalló ${ }^{2}$.

Originalmente, el objetivo de la técnica es determinar las prácticas laborales más y menos efectivas, de modo de proveer hallazgos que fuesen soluciones a problemas prácticos del trabajo (8). Por ejemplo, en enfermería se podría identificar y comprender las conductas claves en enfermeras(os) en su interacción con pacientes o con otros profesionales de la salud o examinar la experiencia de los pacientes en su lugar de hospitalización o su respuesta a un tratamiento. La técnica fue definida por Flanagan como un conjunto de procedimientos para recolectar observaciones directas del comportamiento humano relevante, que facilite la solución de problemas prácticos (8). Lo propio de la Tics es que los entrevistados recuerden y realicen descripciones precisas de eventos relacionados con tareas críticas en su trabajo, no descripciones sobre cómo deben ser hechas las cosas, el interés es con lo real, no el mundo abstracto de la teoría (2).

De acuerdo a lo anterior, se podría plantear que para los profesionales de enfermería esta técnica les permitiría especialmente buscar evidencias más allá del uso de sus impresiones clínicas o basar sus decisiones en hechos anecdóticos. El aporte de la técnica es que las(os) enfermeras(os) puedan realizar un análisis sistemático de los hechos anecdóticos, volviéndose estos últimos en información factible de análisis y relevante en la toma de decisiones (7).

En esta técnica, cuando se hace referencia a un incidente es cualquier actividad humana

\footnotetext{
${ }^{2}$ Su renombrado artículo es considerado uno de los más frecuentemente citados en el campo de la psicología organizacional (Stitt-Gohdes et al. 2000; Butterfield et al. 2000. Ver artículo en: http://psycnet.apa.org/index.cfm?fa=search.displayRecord \&uid=1955-01751-001).
} 
especificable, lo suficientemente completa en sí misma para permitir inferencias y predicciones sobre la persona que realiza una acción (9). Cuando se alude a que el incidente es crítico, se refiere a que el incidente debe ocurrir en una situación en que el propósito o intención de la acción del observado parece lo bastante evidente para el observador y donde sus consecuencias son suficientemente definidas para no dejar duda con respecto a sus efectos (9). En otras palabras, la técnica busca identificar y comprender las conductas extremas (críticas), aquellas que constituyen los determinantes para hablar de un excelente o de un deficiente desempeño de los profesionales de enfermería.

Si bien la técnica en sus inicios se centró en registrar y enumerar episodios sobre conductas que resultan cruciales para desempeñarse en forma eficiente en el trabajo, hoy los procedimientos se complejizan ya que se enfatiza que la conducta siempre ocurre en una situación y, por lo tanto, una adecuada comprensión de la conducta debe considerar el contexto socio-laboral en que ella ocurre (4). Lo anterior implica que, si bien se obtiene información cuantitativa sobre los comportamientos de los integrantes de una organización, no debe sacrificarse el significado, especificidad y validez de la información recabada (10). Lo anterior implica enriquecer la técnica asumiendo una perspectiva fenomenológica que facilite comprender las percepciones y experiencias psicológicas que las personas tienen en sus prácticas laborales (1).

Coherente con una perspectiva fenomenológica, la Tics constituye una herramienta naturalista que obtiene información verbal o escrita desde un entrevistado, sobre un aspecto de su realidad laboral o sobre su experiencia práctica, capturándose una historia en retrospectiva de un evento en la vida de los participantes (11). Desde esta perspectiva, es el entrevistado quien describe la manera en que se produjo la situación y los resultados, por consiguiente, el objetivo de esta técnica sería lograr un entendimiento de los incidentes desde la perspectiva del individuo, teniendo en cuenta tanto los elementos cognitivos, afectivos y conductuales de éste (9).

Pese a que Flanagan enfatizó la búsqueda de objetividad, de exactitud y obtener resultados cuantitativos con su técnica, en un inicio fue escasamente valorada por los investigadores de la época, dado el predominio del enfoque positivista que privilegiaba la experimentación (12). Sin embargo, con el resurgimiento del uso de las metodologías cualitativas en la década del 80 , ha vuelto a ser reconocida y ha comenzado a ser utilizada ampliamente. Se estima que en la actualidad esta técnica debiera ser ubicada en un punto medio dentro del continuo entre metodologías cuantitativas-cualitativas, ya que incorpora elementos de ambas metodologías, pudiéndose clasificar en un diseño mixto (12). O bien, el investigador puede adaptar la técnica al contexto de una metodología de corte cuantitativo o cualitativo. En el caso que la técnica se utilice cuantitativamente se puede evaluar el tipo, naturaleza y frecuencia de los incidentes recolectados (13). Al ser utilizada cualitativamente, la técnica provee datos relativos al discurso del participante, los cuales pueden ser sujetos de análisis y pueden ser codificados y categorizados de acuerdo a los principios de la teoría fundamentada (13). En este caso, incluso se puede llegar a formular una explicación general a partir de un análisis inductivo sistemático. Sin embargo, se debe tener en cuenta que desde sus orígenes la Tics siempre ha tenido sus raíces en la tradición de la investigación fenomenológica, de modo que el análisis cualitativo de los datos se estima central (11). Por último, independiente del marco metodológico de la investigación, esta técnica se utiliza en el contexto de estudios exploratorios que pueden llevar finalmente a proponer hipótesis sobre los fenómenos que se están estudiando (7). 


\section{PASOS EN LA APLICACIÓN DE LA TÉCNICA}

Relativamente pocas modificaciones se han sugerido a la técnica desde hace ya 55 años en que fue introducida y estos cambios serían mínimos en relación al trabajo original de Flanagan (13). Los investigadores concuerdan en describir cinco pasos en la aplicación de esta técnica $(2,5,8,11,14)$.

Primer paso: Es esencial identificar cuidadosamente el objetivo de la conducta a estudiar, debido a la influencia que ésta tiene sobre la posterior recolección y análisis de datos en los siguientes pasos (14). Se debe responder qué es lo que esperan lograr con la investigación y, generalmente, suele ser identificar las conductas específicas positivas y/o negativas con respecto al logro de una actividad en el trabajo (5). Por ejemplo, investigaciones han identificado cuáles eran los factores que influyen en las competencias claves para el éxito en su trabajo en enfermeras supervisoras (15) o identificar las principales dificultades que presentan los pacientes con dolor neurológico crónico en su vida cotidiana (16).

Segundo paso: Se deben planificar tres aspectos claves: a) Determinar los participantes de la investigación, deseable trabajadores familiarizados o, mejor aún, expertos en la actividad estudiada o en la patología de un tipo de pacientes; b) determinar quiénes serán los encuestadores, y c) determinar el tipo de situación a encuestar (determinando la relevancia de la situación en relación al objetivo general). Para asegurar la recolección específica y exacta de los eventos, se recomienda que los incidentes recolectados sean realmente significativos (14). Generalmente, se trata de incidentes atípicos o extremos que son más fáciles de recordar y distinguir (14).

Tercer paso: La recolección de datos se puede realizar de diversas maneras dependiendo de lo acordado en los pasos anteriores, planteándose tres vías principales para obtener los datos: a) Las observaciones directas y grabaciones son útiles cuando se investiga un comportamiento explícito, pero son menos apropiadas para comportamientos encubiertos como las actividades cognitivas, por ejemplo, la planificación o la toma de decisiones; b) los cuestionarios abiertos, además de ser respondidos con lápiz y papel, pueden ser enviados por correo electrónico y remitidos por esta vía, con lo que se puede cubrir una gran cantidad de personas. Una desventaja del uso de cuestionarios es que puede demandar bastante tiempo y trabajo describir adecuadamente los incidentes críticos, disminuyendo la cantidad de cuestionarios devueltos u obteniéndose eventos que no son de utilidad para el estudio (14); c) por último, están las entrevistas individuales o grupales, las que permiten al investigador describir la comunicación no verbal de los participantes e indagar en el relato para lograr mayor profundidad en las respuestas. El formato de entrevista semiestructurada individual se puede considerar como el más apropiado para la recolección de datos, debido a que permite elaborar mejor el relato que otros métodos (14). De hecho, es el formato más utilizado (3). Dada la relevancia de la entrevista individual como método de recolección de datos dentro de la técnica, más adelante se profundizará sobre su desarrollo y aplicación.

Con independencia de la forma de recolección de datos, es posible formular algunas consideraciones metodológicas. $\mathrm{Al}$ igual que en otras técnicas de investigación cualitativa, resulta central una descripción nutrida y amplia de los acontecimientos seleccionados (1). De esta forma, podría evitarse que los datos se entremezclen con opiniones personales, juicios y generalizaciones de los investigadores. Según Flanagan, las observaciones se convierten en una "realidad" (incidentes) cuando una gran cantidad de observadores independientes ofrecen las mismas descripciones de un comportamiento determinado 
(1). De este modo, es fundamental lograr que los hechos sean relatados de un modo lo más objetivo posible y cercano a ellos.

Con respecto al número de incidentes que pueden ser recolectados se ha señalado que lo determinante no es el tamaño de la muestra de participantes, sino el número de incidentes recolectados (11). Ahora bien, el número de incidentes a recolectar no puede ser predefinido sino que al igual que en otras técnicas cualitativas, se deberían recolectar incidentes hasta llegar al punto de saturación, es decir, hasta el momento en que se fracase en recolectar nuevos incidentes $(1,17)$. Probablemente, un total de 10 incidentes es suficiente para un estudio cualitativo de esta índole (15).

Cuarto paso: Muchos investigadores consideran el análisis de datos como el más difícil e importante de la técnica, debido a que puede resultar complejo el clasificar los incidentes. Esta dificultad se agrava por el hecho que no existe una única vía considerada correcta para describir la actividad o experiencia. El propósito de este paso es crear un esquema de categorización que resuma y describa los datos de una manera útil, pero con el menor sacrificio posible de su comprensión, especificidad y validez (5). De hecho, la mayoría de las investigaciones presenta como resultado principal una tabla de frecuencia con las categorías de conductas que se lograron identificar y un posterior análisis de las principales categorías encontradas.

Es imperativo que durante el proceso de análisis se asegure que las interpretaciones no son inferencias. Así, es importante que el investigador no deje que su conocimiento $a$ priori de la situación influya en sus interpretaciones. Si el conocimiento previo es aislado de forma apropiada, se puede obtener un entendimiento más profundo de los relatos y de los argumentos de los participantes (14).

Las categorías principales de clasificación pueden ser deducidas desde los modelos teóricos o formadas sobre la base de la interpretación inductiva (13). Luego corresponde realizar el enlace entre la categoría principal y sus subcategorías en donde se consideran las condiciones en las cuales los eventos ocurrieron, las estrategias adoptadas para tratar con estos incidentes y los resultados posteriores (9). Generalmente, la meta del análisis de contenido es un sistema de clasificación que provee el entendimiento obtenido con respecto a la frecuencia y patrones de los factores que afectan el fenómeno de interés (13).

Una estrategia a considerar dentro de este paso es realizar el análisis temático mediante un análisis inductivo. El proceso de análisis inductivo involucra dos niveles de interpretación (14). El primer nivel de análisis de datos involucra el leer y releer individualmente las transcripciones de los participantes muchas veces para identificar los temas y subtemas. La importancia de que sean leídas individualmente es asegurar que las categorías centrales no sean pasadas por alto. Además, la lectura repetida permite que se descubran inconsistencias y que se encuentren nuevos temas a desarrollar.

El segundo nivel de análisis de datos involucra una lectura comparativa de las transcripciones, lo que vincula segmentos agrupados del texto, entre transcripciones de diferentes eventos. Este análisis comparativo realiza un paralelo entre las diferencias y similitudes de las trascripciones y simultáneamente ayuda al desarrollo de los temas y subtemas (14).

Quinto paso: Interpretación de los datos y reporte de los resultados. El último paso consiste en discutir y reportar los resultados del estudio. Lo primero a realizar en este paso es la revisión de los cuatro pasos anteriores con el fin de determinar la validez y confiabilidad de los datos obtenidos, estableciendo las limitaciones que se han encontrado $(2,11)$. Por ejemplo, señalar si existieron grupos subrepresentados o probabilidades de sesgos de parte de evaluadores En este punto, el investigador es responsable de señalar no sólo las limitaciones, sino que también el grado de 
credibilidad y valor de los resultados finales obtenidos (5).

Finalmente, el informe del estudio o proyecto debe ser retroalimentado a los participantes del estudio de una manera concisa y parsimoniosa para la audiencia, considerando el escaso tiempo que pueden tener algunos profesionales (14).

\section{LA ENTREVISTA DE INCIDENTES CRÍTICOS}

Dada la relevancia que tiene la entrevista en esta técnica, a continuación se describen cuatro aspectos centrales para su puesta en práctica (9):

1) Inicio de la entrevista: Es muy importante crear un ambiente adecuado y hacer un esfuerzo por establecer una relación de confianza con el entrevistado intentando disminuir la tensión o incomodidad que puede dificultar la entrega de información por parte de éste. Por lo general, el investigador comienza explicando brevemente cuál es la naturaleza de la entrevista de incidentes críticos y resume sus propósitos, así como los beneficios que obtendrá la organización mediante el estudio. Luego, se aclaran los aspectos de confidencialidad dando las garantías necesarias al entrevistado.

2) Centrándose en el tema: A los participantes se les pide que recuerden un incidente positivo o negativo señalando (17): a) una descripción detallada del incidente, b) una descripción de las conductas de los involucrados en el incidente y c) los resultados del incidente.

3) Desarrollo de la entrevista: Durante la entrevista se debe solicitar detalles lo más específicos posible sobre la situación. Preguntas claves serían: ¿Qué exactamente sucedió?, ¿Cómo sucedió?, ¿Quiénes participaron?, ¿Qué exactamente hizo usted? y con el fin de establecer la relevancia del incidente, ¿después del incidente, cuáles fueron las consecuencias inmediatas y a largo plazo?

4) Cierre de la entrevista: La entrevista tiende a llegar a un fin naturalmente cuando el entrevistado termina su relato. El entrevistador siempre debe agradecer al entrevistado por su tiempo y la colaboración que se ha empleado, y se debe dejar la impresión de que la entrevista fue de valor. Se puede recordar al encuestado que cualquier revelación será tratada con estricta confidencialidad. Por último, es importante obtener la buena disposición del encuestado para poder volver a entrevistarlo en aspectos específicos de la entrevista en caso de ser necesario.

Sin duda, la entrevista de incidentes críticos no es una herramienta fácil de conducir. Se requiere de un investigador hábil y con competencias específicas que le faciliten obtener respuestas claras del entrevistado, controlando sus propias ideas sobre el asunto y que logre enfocar la entrevista para obtener un relato lo más nítido posible de los eventos. Por ejemplo, de acuerdo a nuestra experiencia, no todos los entrevistados revelarán eventos negativos, por lo que el entrevistador debe ser capaz de indagar y diferenciar entre los sucesos descritos por el entrevistado poniendo énfasis en los que puedan ser realmente significativos.

\section{FORTALEZAS DE LA Tics}

Primero, esta técnica es un procedimiento sistemático e inductivo para recolectar observaciones directas del comportamiento humano. Es sistemático porque permite definir con criterios técnicos aquellos inciden- 
tes que pueden estar afectando el desempeño adecuado de una determinada actividad o influyendo dentro de una situación práctica $(1,18)$. La técnica es inductiva porque los datos recolectados son obtenidos desde la perspectiva del participante y con sus propias palabras. De esta forma, se obtiene una abundante fuente de datos que facilita determinar cuáles incidentes son los más relevantes con respecto al suceso que se está investigando (13). En consecuencia, el empleo de esta técnica puede resultar particularmente útil en los siguientes casos: a) cuando el tema que está siendo investigado ha sido escasamente documentado, b) como un método exploratorio para incrementar el conocimiento acerca de un fenómeno poco conocido o c) cuando es necesario comprender, describir o explicar un fenómeno (13). Por su naturaleza inductiva no requiere una hipótesis y las categorías son formadas a medida que emergen de las respuestas de los participantes, permitiendo al investigador llegar a generar conceptos y modelos explicativos (13).

Segundo, para Flanagan la técnica no consiste en un conjunto rígido de principios a seguir, al contrario, tiene un conjunto de reglas flexibles que pueden ser modificadas para reunir los requerimientos del tema que está siendo estudiado $(2,13)$.

Por último, esta técnica permite que la información pueda obtenerse en forma relativamente rápida. Como se ha visto, la entrevista requiere solamente de respuestas simples, no es preciso que los entrevistados realicen juicios complejos y exhaustivos. Sus respuestas deben ser lo suficientemente detalladas para documentar los hechos. De acuerdo a nuestra experiencia y la de investigadores la duración de estas entrevistas no debería extenderse más allá de 20 minutos (11).

\section{LIMITACIONES DE LA Tics}

Las críticas a la técnica se han centrado en su confiabilidad. La técnica depende de los eventos que son recordados por los participantes y requiere un reporte tan preciso como resulte posible de ellos. Una dificultad puede ser que los participantes pueden no estar acostumbrados o no disponer del tiempo para describir una historia completa cuando se les pide relatar el incidente. Suele resultar una experiencia frecuente obtener sólo una baja tasa de respuestas debido a que esta técnica requiere que los participantes se tomen un tiempo y esfuerzo para describir situaciones con suficiente detalle (13).

Un problema más complejo es que esta técnica al constituir un método de estudio retrospectivo requiere confiar en la memoria del participante. Este punto da origen a la crítica que los recuerdos pueden estar influidos por los sesgos del participante, por ejemplo, recordar sólo lo que se desea recordar o rellenar la información ausente con fabulaciones o el sesgo de autoservicio (11, $13,19)$. Un caso relevante en psicología fue la investigación de F. Herzberg, quien indagó sobre los incidentes críticos que favorecen la motivación laboral; investigadores critican dicho estudio porque los resultados se podrían explicar como reflejo de la tendencia de los participantes a autoatribuirse los éxitos y a culpar al medio ambiente de sus fracasos (19).

Sin embargo, se han señalado varias acciones que pueden ser realizadas para mejorar la confiabilidad de la técnica. Por ejemplo, la posibilidad de realizar más de una entrevista con el mismo sujeto con el fin de completar la información relevante que pueda haber sido pasada por alto. Otra alternativa puede ser completar la información del evento mediante la entrevista a uno de los implicados en el incidente. También se podría repetir la misma entrevista al mismo sujeto con otro entrevistador, lo que daría un índice de confiabilidad interentrevistadores aceptándose sólo aquellas que concuerden entre un 80 a $100 \%$. Además, se puede comparar los resultados de 
las entrevistas con otras fuentes de datos, especialmente aquéllas donde puede existir una evidencia tangible, por ejemplo, un registro que tenga la organización.

Otra fuente de error en la medición puede provenir de la interpretación parcialmente inadecuada que pueden efectuar los entrevistadores a las respuestas dadas por los entrevistados. Se puede evaluar la confiabilidad de esta técnica utilizando la confiabilidad interjueces, es decir, el grado en el cual dos o más jueces pueden estar de acuerdo en una observación dada (13). Lamentablemente, en una revisión de 141 investigaciones que utilizan la Tics se encontró que sólo un $62 \%$ de ellos informan de la confiabilidad interjueces (13). El índice de confiabilidad más utilizado es el coeficiente de concordancia (el número total de concordancias dividido por el número total de decisiones de codificación) y se ha encontrado índices de confiabilidad aceptables, donde el porcentaje del coeficiente de concordancia de 45 estudios es de 85\% (13). En cualquier caso, esta confiabilidad obtenida por dos o más entrevistadores suele ser el producto de una inteligibilidad intersubjetiva, es decir, un cierto nivel de acuerdo o convención entre los entrevistadores, lo que facilita su concordancia. En la práctica estos hallazgos sugieren la necesidad de un entrenamiento previo común y de ensayos de práctica entre los entrevistadores antes de iniciar el estudio.

Finalmente, se estima que ampliar el número de jueces utilizados para categorizar los datos de la técnica es una estrategia valiosa para abordar el problema planteado previamente (13). De hecho, la mayoría de los estudios que utilizan la técnica reportan dos o tres jueces, quienes analizan y categorizan los incidentes críticos. El número de jueces a través de todos los estudios varía desde 1 hasta 8 , con una media de 2,6 jueces (13).

\section{APLICACIONES DE LA TÉCNICA DE INCIDENTES CRÍTICOS EN EL CONTEXTO DE ENFERMERÍA}

Se puede distinguir 3 amplias áreas de aplicación de la técnica en enfermería (2). Primero, e históricamente la más utilizada, gestión y administración. La técnica tiene el potencial de mejorar el desempeño laboral ya que su método puede revelar las conductas claves asociadas al desempeño excelente. Por ejemplo, se le puede pedir a expertos (profesores experimentados) que identifiquen las conductas claves en situaciones críticas o cómo resuelven los problemas más importantes en el trabajo (6). También se puede pedir que identifiquen las conductas deficitarias en el trabajo y así identificar necesidades de capacitación de los profesionales de enfermería.

Además, la Tics es una herramienta práctica en programas de desarrollo organizacional para identificar problemas de funcionamiento de los servicios de salud (20). Específicamente, se podría pedir a funcionarios de la salud que identifiquen los problemas más importantes que se producen en el funcionamiento de la unidad de trabajo, desglosando en el análisis que llevó a esta situación, cómo se actuó en el momento y cuáles fueron las consecuencias. Luego de realizar varias entrevistas, probablemente será posible encontrar interesantes patrones en las respuestas dadas por los participantes.

Cabe destacar que la Tics puede resultar central para la realización de una evaluación de competencias laborales de los profesionales de enfermería $(15,21)$. Un desarrollo de la técnica ha sido proponer que la Tics puede ser la base para construir escalas de evaluación ancladas en comportamientos, su nombre en inglés BARS $^{3}$ (22). En esencia, las BARS toman la información cualitativa que resulta de la Tics y la lleva al plano de lo cuantitativo, al priorizar los comportamientos en una escala. Esta técnica es una herramienta

${ }^{3}$ Behaviourally Anchored Rating Scales. 
valorada en el modelo de administración por competencias ya que se requiere identificar las competencias requeridas para un desempeño superior, pudiéndose desarrollar escalas de evaluación que pueden ser utilizadas (6). Por otra parte, quien desarrolló el concepto de competencias laborales, David McClelland, creó para evaluarlas la entrevista de eventos conductuales, que es, según el autor, una adaptación de la entrevista de incidentes críticos $(9,23)$.

En segundo lugar, está su aplicación en educación en enfermería. De especial importancia para el desarrollo profesional está el uso para facilitar la práctica reflexiva en los trabajadores sobre su quehacer. Por ejemplo, se puede pedir a los profesionales que identifiquen las situaciones que inciden significativamente en su desarrollo profesional, e incluso reflexionen acerca de dichas experiencias. Otro ejemplo, solicitar a docentes de enfermería que reflexionen sobre cuáles situaciones inciden en la construcción del conocimiento práctico de sus estudiantes y así conocer el significado que tiene para ellos la experiencia de aprendizaje (1). Otro ejemplo sería identificar los factores que influyen positiva o negativamente durante el proceso de enseñanza-aprendizaje de los estudiantes de enfermería (24).

Se sostiene que la Tics es capaz de ayudar a generar en los participantes una mayor comprensión de las situaciones vividas, ya que los participantes pueden llegar a conectarse íntimamente con su experiencia personal y pueden pasar de la descripción de hechos específicos a una comprensión más general de sus supuestos y creencias sobre los hechos, lo que proporcionaría un medio poderoso para estimular un aprendizaje transformativo en los trabajadores participantes del estudio (11). Es decir, si se aplica a profesionales de enfermería la técnica puede estimular a que ellos realicen una reflexión sobre incidentes críticos de su propio trabajo y aprendan de su propia experiencia (7). De acuerdo a nuestra experiencia, al recordar incidentes críticos, se revive la experiencia inclusive con la emoción $y$, en ese contexto, puede llegar a producirse reestructuración cognitiva que muestre aspectos no considerados de sus prácticas y entregue recursos cognitivos nuevos para abordar situaciones similares en el futuro.

Una tercera aplicación correspondería a su uso en el tratamiento de pacientes, por ejemplo se puede entrevistar a pacientes con respecto a hechos relevantes de su evolución (16).

\section{CONSIDERACIONES FINALES}

Aunque podría pensarse que no resulta de interés poner atención a una técnica de investigación creada hace ya más de 55 años, la presente revisión bibliográfica muestra que su utilidad y efectividad se mantiene vigente. Por consiguiente, su uso puede continuar, dado que sus principales características (relativa sencillez de aplicación y capacidad para encontrar categorías analizables) pueden ser relevantes para la realización, especialmente de evaluaciones de competencia laborales y para la realización de una práctica reflexiva destinada a encontrar oportunidades de mejora en el trabajo de enfermería.

Dado que la técnica ofrece una forma sistemática, contextualizada y flexible de recolección de datos pudiéndose aplicar efectivamente en un amplio rango de situaciones en enfermería, la Tics proporciona una interesante perspectiva de los comportamientos de los integrantes de los centros de salud que facilita comprender los obstáculos que éstos enfrentan y puede proporcionar guías para efectuar potenciales mejoramientos.

\section{REFERENCIAS}

1. Noreña A, Cibanal L. La técnica del incidente crítico y sus implicaciones en el 
desarrollo de la investigación en enfermería. Index Enferm 2008; 17(1):48-52.

2. Bradbury-Jones C, Tranter S. Inconsistent use of the critical incident technique in nursing research. J Adv Nurs. 2008; 64(4):399-407.

3. Angelides P. The development of an efficient technique for collecting and analyzing qualitative data: the analysis of critical incidents. Qualitative Studies in Education. 2001; 14(3):429-42.

4. Stitt-Gohdes W, Lambrecht J, Redmann D. The critical-incident technique in job behavior research. Journal of Vocational Education Research 2000; 25(1):63-77

5. Butterfield L, Borgen W, Amundson N, Maglio A. Fifty years of the critical incident technique: 1954-2004 and beyond. Qualitative Research. 2004; 5(4):475-97

6. Marrelli A. The performance technologist's toolbox: Critical incidents. Performance Improvement. 2005; 44(10):40-4.

7. FitzGerald K, Seale N, Kerins C, McElvaney $\mathrm{R}$. The critical incident technique: a useful tool for conducting qualitative research. J Dent Educ. 2007;72(3):299-304.

8. Flanagan J. The critical incident technique. Psychol Bull 1954; 15(4): 327-58.

9. Chell E. Critical incident technique. En: Cassell C, ed. Essential Guide to Qualitative Methods in Organizational Research. Londres: Thousand Oaks; 2004. p. 45-60.

10. Rodríguez M. Ética gerencial: Comportamientos éticos de los gerentes que más valoran los empleados en Colombia. Revista Universidad EAFIT. 2006; 42(143):41-54.

11. Sharoff L. Critique of the critical incident technique. J Res Nurs. 2007; 12(6):1-10.

12. Bedi R, Davis M, Williams M. Critical incidents in the formation of the therapeutic alliance from the client's perspective. Psychotherapy. 2005; 42(3):311-23.

13. Gremler D. The critical incident technique in service research. Journal of Service Research. 2004;7(1): 65-89.

14. Schluter J, Seaton P, Chaboyer W. Critical incident technique: a user's guide for nurse researchers. J Adv Nurs. 2008;
61(1):107-14.

15. Arvidsson B, Fridlund B. Factors influencing nurse supervisor competence: a critical incident analysis study. J Nurs Manag. 2005; 13:231-7.

16. Hensing G, Sverker A, Leijon G. Experienced dilemmas of everyday life in chronic neuropathic pain patients - results from a critical incident study. Scand J Caring Sci. 2007; 21(2):147-54.

17. Victoroff K, Hogan S. Students' perceptions of effective learning experiences in dental school: A qualitative study using a critical incident technique. J Dent Educ. 2006; 70(2):124-32.

18. Keatinge D. Versatility and flexibility: Attributes of the critical incident technique in nursing research. Nurs Health Sci. 2002; 4(1-2):33-9.

19. Sachau D. Resurrecting the MotivationHygiene Theory: Herzberg and the positive psychology movement. Human Resource Development Review. 2007; 6(4):377-93.

20. Davis P. Critical incident technique: a learning intervention for organizational problem solving. Development and Learning in Organization. 2006; 20(2):13-16.

21. Lewis R, Yarker J, Donaldson-Feilder E, Flaxman P, Munir F. Using a competency-based approach to identify the management behaviours required to manage workplace stress in nursing: A critical incident study. Int J Nurs Stud. 2010;47(3):307-13.

22. Rodríguez M, Bustamante U. Desarrollo de competencias para el comportamiento ético-gerencial: Un enfoque de responsabilidad. Cuad. Adm. Bogotá (Colombia). 2008; 21(35):205-28.

23. McClelland D. Identifying competencies with behavioral-event interviews. Psychol Sci 1998; 9 (5):331-9.

24. Zani A, Nogueira M. Critical incidents in the teaching-learning process of a nursing course through the perception of students and faculty. Rev Lat Am Enfermagem. 2006; 14:742-8. 